\title{
2 Tatsächliche Grundlagen und Materialien
}



Die im Rahmen dieser Expertise zur Rede stehenden Proben werden in ganz unterschiedlichen Zusammenhängen gewonnen. Aus diesem Grunde ist der Kreis der von der Probengewinnung Betroffenen ebenfalls recht heterogen. Auch können deshalb für die zu beurteilende Rechtslage ganz unterschiedliche Rechtsgrundlagen relevant werden.

Im Folgenden sollen daher die der Expertise zugrunde gelegten Szenarien der Probengewinnung inklusive der spezifischen Vorgaben für die juristische Aufarbeitung kurz vorgestellt werden. Im Anschluss daran werden die Materialien aufgeführt, die hinsichtlich der zu beurteilenden Tatsachen- und/oder Rechtsbasis für die Expertise zu berücksichtigen waren.

\subsection{Szenarien}

Für die Erstellung der juristischen Expertise wird von folgenden Szenarien ausgegangen.

\section{Szenario 1: Lebensmittelprobe aus dem Handel}

Hier werden Proben im Lebensmittelhandel käuflich erworben. Aus diesen Proben werden sodann Isolate generiert, die in Verbindung mit Informationen zur Art des Lebensmittels und dessen Herkunft (Händlertyp, gegebenenfalls Zuordnung zum landwirtschaftlichen Betrieb, Angaben zur Region etc.) ausgewertet und bewertet werden.

Für dieses Szenario wird unten in Kapitel 6 keine Forschungsinformation und keine Einwilligungserklärung formuliert, weil der jeweilige Wissenschaftler/ das jeweilige Institut beim kaufweisen Erwerb der Proben ohne Weiteres Eigentümer der Proben wird. Stattdessen soll für dieses Szenario das Haftungsrisiko diskutiert werden, das sich aus möglichen Pflichtverletzungen von Wissenschaftlern (etwa der Verletzung von Anzeige- und Meldepflichten aus tierseuchenrechtlichen Regelungen) und aus Produkthaftungsregelungen ergibt, welche jedoch grundsätzlich die Lebensmittelhersteller treffen.

\section{Szenario 2: Probenentnahme in einem Betrieb}

Einzeltier- oder Sammelproben (von einer Tiergruppe) werden bei dieser Art der Probengewinnung in einem Betrieb (landwirtschaftlicher Betrieb, Tierhändler, Teichwirtschaft, Pferdehaltung, Gehegewild, Zoo, Bienenhaltung u.ä.) gesammelt und im Labor bearbeitet. Neben der Information zur Probe (Tag, Lokalisation und Art der Probenentnahme) werden dabei auch Informationen zum Betrieb und gegebenenfalls zum Vorbesitzer der Tiere erfasst. Hierzu dienen eigene Erfassungsinstrumente der beteiligten Forschergruppen(Fragebogen, Beobachtungen, Messungen etc.) oder auch die Nutzung von betrieblichen Daten, die an anderen Stellen bereits erfasst worden sind (Stallbuch, Betriebssoftware, HI-Tier ${ }^{1}$ ).

1 Herkunftssicherungs- und Informationssystem für Tiere, www.hi-tier.de 
Nach Abstimmung mit dem Auftraggeber dieser Expertise sind bei diesem Szenario Mustertexte für folgende Möglichkeiten zu entwickeln:

1. Einwilligungserklärung mit Ausschluss von bestimmten Untersuchungen (alle anzeigepflichtigen Tierseuchen);

2. Einwilligungserklärung zur Untersuchung auf anzeigepflichtige Tierseuchen mit Daten zum groben geografischen Raster, wobei eine Anonymisierung als Selbstverpflichtung durch den Wissenschaftler zugesichert wird;

3. Einwilligungserklärung mit einer Pseudonymisierung auf Betriebsebene.

Alle drei Möglichkeiten können mit

- einer Eigentumsübertragung bzw.

- einer Übertragung des Nutzungsrechts

an der Probe kombiniert werden.

Bei einer Übertragung des Nutzungsrechts sollen sämtliche Ergebnisse, Isolate und Gewinne aus der Forschung miteingeschlossen werden.

Ergänzend sind rechtliche Besonderheiten darzustellen, die sich daraus ergeben, dass Proben von Tieren gewonnen werden, die nicht der Lebensmittelgewinnung dienen (Haustiere) und von Privatpersonen gehalten werden.

Weiter sind im Kontext dieses Szenarios folgende Einzelfragen zu beantworten:

- Inwiefern spielen die Rechte des Betriebs bzw. des Betriebsinhabers bzw. die allgemeinen Persönlichkeitsrechte des Betriebsleiters eine Rolle bei der Verwendung der tierischen Proben?

- Wie sehen die Haftungsrisiken bzw. Konsequenzen für Wissenschaftler aus?

- Ist ein Ausschluss des Widerrufs der Einwilligungserklärung zulässig?

\section{Szenario 3: Probenentnahme im Schlachthof}

Proben werden im Schlachthof als individuelle Probe vor, während oder nach der Schlachtung erfasst (beispielsweise Fleischsaft, Blut, Organproben). Neben der Information zur Probe (Tag, Lokalisation und Art der Probenentnahme) werden Informationen zum Betrieb durch Nutzung von die Schlachtung begleitenden Dokumenten gesammelt. Zudem sollen betriebliche Daten genutzt werden, die am Schlachthof oder an anderen Stellen bereits erfasst sind (Stallbuch, Betriebssoftware, HI-Tier).

Zu diesem Szenario ist festgestellt worden, dass keine großen Unterschiede zum Szenario 2 bestehen, weil Schlachthöfe die Tiere ankaufen und damit - mit Ausnahmen - als Tiereigentümer anzusehen sind. Insofern sind zu diesem Szenario lediglich die von Szenario 2 abweichenden Besonderheiten darzustellen und im Übrigen Mustertexte mit den gleichen Optionen zu erstellen wie für Szenario 2. 


\section{Szenario 4: Probenentnahme von Wildtieren}

a. Lebendfang:Wildtiere werden in diesen Fällen gefangen, betäubt, beprobt und wieder freigelassen. Dabei müssen Arten- und Naturschutzbestimmungen berücksichtigt werden.

b. Proben aus der Jagdstrecke: Jagdbares Wild wird hierbei vom Jagdausübungsberechtigten erlegt und anschließend beprobt. Daten zum Erlegen und zum Erlegungsort werden erfasst.

Bei diesem Szenario sind folgende Besonderheiten maßgeblich zu berücksichtigen:

- Wildtiere sind nach $₫ 960 \mathrm{BGB}^{2}$ herrenlos und haben keinen Eigentümer, der eine Einwilligungserklärung abgeben könnte.

- Jagdausübungsberechtigte besitzen jedoch ein Aneignungsrecht ( $\mathbb{1}$ Abs. 5 BundesjagdC ${ }^{3}$ ) für alle Tierarten, die dem Jagdrecht unterliegen ( $\$ 2$ BundesjagdC); die Einwilligung ist deshalb in der Regel von dem jeweiligen Jagdausübungsberechtigten einzuholen.

- Bei nicht dem Jagdrecht unterliegenden Tieren greift das Bundesnaturschutzgesetz ${ }^{4}$, und zwar sowohl für nicht besonders zu schützende Arten ( $\$ 39$ Abs. 1 Nr. 1 i.V.m. $\$ 67$ Abs. 1 BNatSchG) als auch für besonders

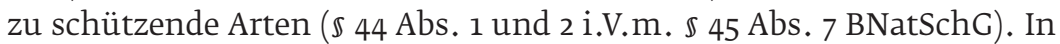
beiden Fällen ist die nach Landesrecht für Naturschutz und Landschaftspflege zuständige Behörde (= untere Naturschutzbehörde) vor der Probenerhebung zu kontaktieren.

- Bei Wildtieren (beispielsweise der Storch auf einem Stalldach, Fledermäuse in einer Zitadelle) muss die Zugangserlaubnis des Crundstückseigentümers eingeholt werden. Dies sollte dokumentiert werden und kann mit einer Forschungsinformation und Einwilligungserklärung verknüpft werden.

\subsection{Materialien}

Den Bearbeitern dieser Expertise wurde seitens des Auftraggebers das Muster einer Forschungsinformation und Einwilligungserklärung (Stand: 03. März 2009) zur Verfügung gestellt. Ferner lagen ihnen zur Erläuterung der relevanten Sachverhalte die Protokolle der Sitzung der Arbeitsgruppe Zoonosen und Infektionsforschung der TMF vom 26. Mai 2009 und des Kick-off-Meetings zu dieser Expertise vom 13. Januar 2010 (nebst Szenarien und Fragen entsprechend der Diskussion zu diesem) vor, ferner das Dokument „Ergänzungen der Prob-

2 Bürgerliches Gesetzbuch in der Fassung der Bekanntmachung vom 02. Januar 2002, BGBI I S. 42.

3 Bundesjagdgesetz in der Fassung der Bekanntmachung vom 29. September 1976, BGBI I S. 2849.

4 Gesetz über Naturschutz und Landschaftspflege (BNatSchG) vom 29. Juli 2009, BGBI I S. 2542. 
lemskizze“ vom 26. Oktober 2009. Am genannten Kick-off-Meeting konnte einer der Bearbeiter dieser Expertise zudem persönlich teilnehmen.

Weiterhin wurden die Publikationen „Biomaterialien - Rechtliche Rahmenbedingungen“ (Simon et al. 2006, TMF-Schriftenreihe Band 2) und „Checkliste und Leitfaden zur Patienteneinwilligung“ (Harnischmacher et al. 2006, TMF-Schriftenreihe Band 3) herangezogen, um die Übertragbarkeit von Ergebnissen früherer Untersuchungen im Zusammenhang mit menschlichen Proben auf die vorliegend behandelte Thematik zu überprüfen.

Ferner hatte einer der Bearbeiter dieser Expertise Gelegenheit zur Teilnahme am Nationalen Symposium für Zoonosenforschung am 06./07. Oktober 2011 in Berlin. 